MS31 Crystal energy

landscapes: computation and

uses

Chairs: Anthony Reilly, Marcus Neumann

\section{MS31-P1 Quantifying \\ Intermolecular Interaction Energies in Organic Clathrates at High Pressure}

Espen Eikeland ${ }^{1}$, Solveig R. Madsen ${ }^{1}$, Maja K. Thomsen ${ }^{1}$, Jacob Overgaard $^{1}$, Mark A. Spackman ${ }^{2}$, Bo B. Iversen ${ }^{1}$

1. Center for Materials Crystallography, Department of Chemistry and iNANO, Aarhus University, DK-8000 Aarhus C, Denmark

2. School of Chemistry and Biochemistry, M310 University of Western Australia, 35 Stirling Hwy, Crawley, 6009, Australia

email: eikeland@chem.au.dk

Molecular self-assembly is at the heart of the field of crystal engineering and supramolecular chemistry. Yet, it is striking that the fundamental chemistry governing even simple self-assembly processes are not well understood. To improve our understanding it is necessary to precisely quantify the different superimposed intermolecular interactions, such as dispersion and electrostatic energies. In this project novel energy frameworks [1] are used to study and eloquently illustrate host-guest interaction energies in three different hydroquinone clathrate systems containing formic acid, acetonitrile and methanol respectively, as the different guest molecules. The intermolecular interaction in these systems have been probed by subjecting single crystals to high external pressure, forcing the molecules closer together. In this way it is possible to observe how changing the guest molecule affect the compression of the host structure. For the hydroquinone - formic acid structure an interesting new pressure-medium-dependent phase transition is observed where the host structure collapse and the guest molecules migrate out of the cavities. The phase transition can be kinetically hindered using a non-hydrostatic pressure-transmitting medium, enabling the comparison of intermolecular energies in two polymorphic structures in the same pressure range [2]. For the hydroquinone - acetonitrile system the guest molecule is found to heavily influence the compression of the host structure until a phase transformation occurs above $4 \mathrm{GPa}$, where the guest molecule break the host symmetry, reducing the space group symmetry to P-1. Energy frameworks can be used to explain the nature of the phase transformations. A new analysis method combining void space analysis and equation of state calculations have been used to quantify the compression of the host cavities and how they are influenced by the presence of different guest molecules. In this way we show that in the hydroquinone - formic acid clathrate system the cavity volume is reduced by more than $50 \%$ up to $4 \mathrm{GPa}[2]$.
[1] M. J. Turner, S. P. Thomas, M. W. Shi, D. Jayatilaka, M. A. Spackman. Chem. Commun. 2015, 51, 3735- 3738

[2] E. Eikeland, M. K. Thomsen, S. R. Madsen, J. Overgaard, M. A. Spackman, B. B. Iversen. Structural Collapse of the Hydroquinone-Formic Acid Clathrate: A Pressure-Medium-Dependent Phase Transition Chem. Eur. J. 2016, 22, 4061 - 4069

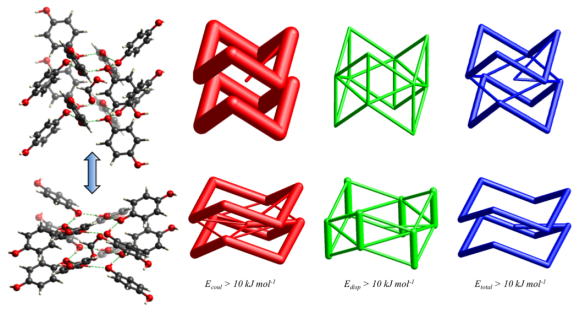

Figure 1. Energy frameworks for the hydroquinone - formic acid clathrate before (top row) and after (bottom row) the structural collapse of the host structure at $4 \mathrm{GPa}$. The electrostatic, dispersion and total interaction energies are colored red, green and blue, respectively.

Keywords: Energy frameworks, intermolecular energies, high-pressure crystallography, host-guest interactions, organic clathrates 